\title{
Enhanced contribution to quark and neutron electric dipole moments with small mixing of right-handed currents and CKM CP violation
}

\author{
Yi Liao \\ Department of Modern Applied Physics, Tsinghua University, Beijing 100084, People's Republic of China \\ Xiaoyuan $\mathrm{Li}$ \\ Institute of Theoretical Physics, The Chinese Academy of Sciences, Beijing 100080, People's Republic of China
}

(Received 1 July 1999; published 6 March 2000)

\begin{abstract}
We study the light quark and the neutron electric dipole moments (EDM's) under the assumptions that the $C P$ source is still in the usual CKM matrix and that there is a small mixing of right-handed charged currents in the quark sector. We find that the EDM's arise already at two-loop order that are much larger than the standard model result even for a small mixing.
\end{abstract}

PACS number(s): 11.30.Er, 12.60.Cn, 13.40.Em

One of the outstanding questions that remains unanswered in present day particle physics is the origin of $C P$ violation [1]. Even 35 years after its discovery, our knowledge is still mainly limited to the neutral kaon system. However, to acheive a consistent picture of $C P$ violation, it is extremely desirable to observe its effects in other systems. In relativistic quantum field theories, a $C P$-violating interaction can induce $P$ and $T$ violating electric dipole moments (EDM's) for elementary particles. So, one promising possibility beyond the kaon system is provided by the observation of the neutron and the electron EDM's. The current experimental bounds on them are, respectively, $|d(e)|<4.3 \times 10^{-27} e \mathrm{~cm}$ [2], $|d(n)|<1.1 \times 10^{-25} e \mathrm{~cm}$ [3]. Recently, the bound on $d(n)$ has been pushed to $6.3 \times 10^{-26} e \mathrm{~cm}[4]$ and significant improvement is expected to be available in the near future $[5]$.

Because of its fundamental importance and experimental progress there has been continuing theoretic interest in searching for mechanisms to induce EDM's. In the standard model (SM) of electroweak interactions, the only $C P$-violating parameter is the $C P$ phase in the CabibboKobayashi-Maskawa (CKM) matrix of the quark left-handed charged currents [6]. There is no contribution to quark (and thus neutron) EDM's at one-loop order because the relevant amplitude is proportional to the moduli of the CKM matrix. Naively, the first contribution should come from the twoloop amplitude that is rich enough in flavor structure. However, as first pointed out by Shabalin [7] and confirmed afterwards by others [8-10], the two-loop contribution to quark EDM's actually vanishes strictly. The same null result was also witnessed in the $W^{ \pm}$EDM $[11,12]$. This is surprising in the sense that there is no symmetry which would demand the vanishing of EDM's at the lowest nontrivial order. Actually, when QCD is turned on, quark EDM's already arise at three-loop order [13-15]. The numerical result is indeed too small to be observable in the near future. It seems, therefore, that the CKM mechanism of $C P$ violation would be irrelevant to an experimental search for EDM's, although there has been no clear reason why this must be so. Beyond the SM, many new possibilities are open to $C P$ violation. Generally, the EDM's are induced at one-loop order that usually turns out to be too large. As a result, some degree of fine tuning is necessary to suppress these contributions. For instance, in the minimal supersymmetric standard model, one has to appeal to assumptions about the size of $C P$ phases [16] and the spectrum [17], or the cancellation among different contributions [18]. In this regard, EDM's should most naturally arise at two-loop order.

In this note, we shall stick to the more conservative possibility that $C P$ violation is still restricted to the CKM matrix as in the SM but a small mixing of right-handed charged currents is allowed in the quark sector. This consideration is partly motivated by the recent result on $\epsilon^{\prime} / \epsilon$ [19] which indicates that we should surely take the CKM mechanism seriously. We would like to investigate how far we can go with only CKM $C P$ violation. We observe that with mixed left- and right-handed charged currents the chirality flip required by the EDM operator is not necessarily to be implemented by a small external quark mass but could be done by an internal quark mass. It is then possible to obtain terms that are not suppressed by external quark masses and thus gain an enhancement in EDM's as compared to the SM case. Theoretically, the model considered here may arise, for example, as a special case of left-right symmetric models [20] in which the only the $C P$ source is assumed to be in the usual CKM matrix. These models have received heated attention [21] due to recent experimental evidence for neutrino oscillations [22] and a possible anomaly in the $Z \rightarrow b \bar{b}$ decay [23].

We consider the light quark EDM's from which the neutron EDM is constructed by using the $\mathrm{SU}(6)$ relation

$$
d(n)=\frac{4}{3} d(d)-\frac{1}{3} d(u)
$$

Possible enhancements from long distance physics will not be considered here [24]. The effective Lagrangian for the EDM interaction is defined as

$$
\mathcal{L}_{\mathrm{eff}}=-\frac{i}{2} d \bar{\psi} \gamma_{5} \sigma_{\mu \nu} \psi F^{\mu \nu}
$$

where $F^{\mu \nu}$ is the electromagnetic tensor and $d$ is the EDM of the fermion $\psi$. As mentioned previously, the $C P$-violating interaction is assumed to be 


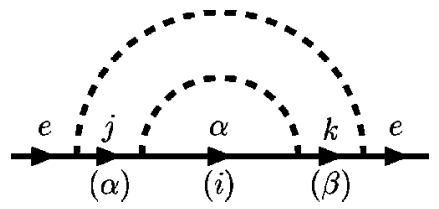

FIG. 1. The Feynman diagram that contributes to the EDM of the up-type quark $u_{e}$. A background electromagnetic field is understood to be attached to internal lines in all possible ways. The dashed lines represent $W^{ \pm}$and $G^{ \pm}$bosons. The diagram for the down-type quark $d_{e}$ is obtained by substitutions: $\alpha \rightarrow i$ and $j, k$ $\rightarrow \alpha, \beta$.

$$
\mathcal{L}_{W}=\frac{g}{\sqrt{2}} \sum_{\alpha, i} V_{\alpha i} \bar{u}_{\alpha} \gamma^{\mu}\left(P_{L} c_{\theta}+P_{R} s_{\theta}\right) d_{i} W_{\mu}^{+}+\text {H.c. }
$$

Here $P_{L}=\frac{1}{2}\left(1-\gamma_{5}\right), P_{R}=\frac{1}{2}\left(1+\gamma_{5}\right)$ and the Greek and Latin letters denote, respectively, the up- and down-type quarks. $V_{\alpha i}$ is the entry $(\alpha, i)$ of the CKM matrix. $c_{\theta}$ $=\cos \theta, s_{\theta}=\sin \theta$, where $\theta$ is the mixing angle of the leftright gauge bosons $W_{L, R}^{ \pm}$, and $W^{ \pm}$is to be identified as the usual charged weak boson. The mixing angle is constrained by low-energy processes to be $|\theta|<10^{-2} \sim 10^{-3}$ [25]. We stress again that we have assumed that the only $C P$ source is the single phase in the usual CKM matrix. If this is not the case, EDM's will generally be induced at one-loop order due to the mismatch between two CKM matrices in the left- and right-handed currents and due to the new phase in the $W_{L, R}^{ \pm}$ mixing [26,27]. We have also safely neglected contributions from extra heavier charged gauge bosons. Since we work in renormalizable gauges, we need to specify the interaction Lagrangian for the would-be Goldstone bosons $G^{ \pm}$. The easiest way to do so is by examining the cancellation of the gauge parameter dependence between $W^{ \pm}$and $G^{ \pm}$contributions to a physical process, e.g., $\bar{u}_{1} d_{1} \rightarrow \bar{u}_{2} d_{2}$. We find

$$
\begin{aligned}
\mathcal{L}_{G}= & \frac{g}{\sqrt{2}} \frac{1}{m_{W}} \sum_{\alpha, i} V_{\alpha i} \bar{u}_{\alpha}\left[c_{\theta}\left(m_{\alpha} P_{L}-m_{i} P_{R}\right)\right. \\
& \left.+s_{\theta}\left(m_{\alpha} P_{R}-m_{i} P_{L}\right)\right] d_{i} G^{+}+\text {H.c. }
\end{aligned}
$$

To simplify the computation of diagrams involving $W^{ \pm}$exchange, we shall use the background field [28-30] (or the nonlinear [31]) $R_{\xi}$ gauge with $\xi=1$. The relevant $W^{+} W^{-} A$ vertex ( $A$ is the background electromagnetic field) is given in Ref. [10].

The Feynman diagram that contributes to the quark EDM's is shown in Fig. 1. There are four groups of contributions, namely $W G, G W, W W$, and $G G$, where the first and second letters refer to the bosons exchanged in the outer and inner loops, respectively. The final $\gamma_{5}$ in the effective EDM operator can only come from vertices since there would be no $P$ violation if no $\gamma_{5}$ were involved in these vertices. Details concerning renormalization and calculational techniques will not be presented here. We refer the interested reader to Ref. [10] although completely new terms will appear in the current case as a consequence of the leftright mixing. In that work, we studied possible contributions to the quark EDM's arising from charged Higgs bosons in the two Higgs doublet model with only CKM $C P$ violation,

$$
\mathcal{L}_{H}=\frac{g}{\sqrt{2}} \frac{1}{m_{W}} \sum_{\alpha, i} V_{\alpha i} \bar{u}_{\alpha}\left(C_{\alpha i}+C_{\alpha i}^{\prime} \gamma_{5}\right) d_{i} H^{+}+\text {H.c. },
$$

where $C_{\alpha i}$ and $C_{\alpha i}^{\prime}$ are real couplings depending on the masses of $u_{\alpha}$ and $d_{i}$. We found that the contribution to the EDM of the quark $u_{e}$ has a separate form:

$$
d\left(u_{e}\right)=\operatorname{Im}\left(V_{e k} V_{\alpha k}^{*} V_{\alpha j} V_{e j}^{*}\right)\left[H\left(m_{k}\right)-H\left(m_{j}\right)\right],
$$

if $C_{\alpha i}$ and $C_{\alpha i}^{\prime}$ are related by the following relations:

$$
C_{\alpha i}=x m_{i}+y m_{\alpha}, \quad C_{\alpha i}^{\prime}=x m_{i}-y m_{\alpha} .
$$

Here $H$ is also a function of the masses of bosons, $u_{\alpha}$ and $u_{e}$, but the crucial point is that it depends exclusively on $m_{k}$ or $m_{j} . x$ and $y$ are mass-independent constants that depend on the detail of the model. It is this separate structure that leads to the complete cancellation when summation over internal flavors is taken and when the unitarity of the CKM matrix is assumed. We can see from Eq. (4) that the above relations are not respected any longer when the left-right mixing is introduced. Therefore, we shall probably obtain a nonvanishing result in the current case.

Our result should depend on $c_{\theta}$ and $s_{\theta}$ to the fourth power. If the charged current is purely left-handed $\left(s_{\theta}=0\right)$, we return to the vanishing result in the SM. The purely righthanded case $\left(c_{\theta}=0\right)$ is related by a parity reflection, i.e., by reversing the sign of EDM. If the current is purely vectorial $\left(c_{\theta}=s_{\theta}\right)$ or purely axial-vectorial $\left(c_{\theta}=-s_{\theta}\right)$, we still arrive at a vanishing result. Therefore, the final, possibly nonvanishing result should be proportional to $\left(c_{\theta}^{2}-s_{\theta}^{2}\right) c_{\theta} s_{\theta}$. Let us now examine qualitatively what quark mass dependence should be expected. The $C P$ source is in the CKM matrix which arises from diagonalization of quark mass matrices. If any two up-type or down-type quarks are degenerate, there will be no $C P$ violation and thus no EDM's. This implies, for example, that the contribution to $d\left(u_{e}\right)$ from Fig. 1 must be antisymmetric in $m_{j}$ and $m_{k}$. Hence the pair of diagrams related by mirror reflection $j \leftrightarrow k$ contribute the same to $d\left(u_{e}\right)$ which is proportional to $\operatorname{Im}\left(V_{e k} V_{\alpha k}^{*} V_{\alpha j} V_{e j}^{*}\right)$ while the $\operatorname{Re}\left(V_{e k} V_{\alpha k}^{*} V_{\alpha j} V_{e j}^{*}\right)$ part is cancelled as required by hermiticity [9]. To proceed further, we make use of the hierarchical structure in quark masses, $m_{t} \gg m_{W} \gg m_{b} \gg m_{c} \gg m_{s} \gg m_{u, d}$. At the moment, all quarks except the top are treated as light on the same footing as compared to the reference scale $m_{W}$. We shall be concerned with the first nontrivial terms which are of lowest order in light quark masses. Higher order terms are safely ignored even if they are possibly enhanced by factors of $m_{t}^{2}$ since $\left(m_{q}^{2} / m_{W}^{2}\right)\left(m_{t}^{2} / m_{W}^{2}\right) \ll 1$, where $q$ can be any quark except the top. The external quark mass $m_{u}$ or $m_{d}$ is also ignored from the beginning. Then, the chirality flip is to be made by internal quark masses so that the result depends on them to some odd power. We first consider the contribution to the EDM of the $u$ quark, $d(u)$. In this case, $u_{\alpha}$ can be heavy $(t)$ or light $(c)$ but $d_{j}$ and $d_{k}$ are always light. Because of the unitarity of the CKM matrix, the de- 
sired terms must involve $d_{j}$ and $d_{k}$ masses simultaneously in order to survive the summation over the flavor pair $(j k)$. Therefore, we expect $d(u) \propto m_{j} m_{k}\left(m_{j}-m_{k}\right)$ up to logarithms associated with each term. For the EDM of the $d$ quark, we should first discriminate two cases; namely, the quarks $u_{\alpha}$ and $u_{\beta}$ are both light $(u, c)$ or one of them is heavy $(t)$. Since $d_{i}$ is always light, the desired terms must involve its mass; otherwise they will be cancelled upon summing over the flavor $i$. Therefore, if $u_{\alpha}$ and $u_{\beta}$ are both light as well, we expect this part of the contribution to be proportional to $m_{\alpha} m_{\beta}\left(m_{\alpha}-m_{\beta}\right) m_{i}^{2}$ so that it can be ignored. Now suppose $u_{\alpha}$ is the heavy top. Although the amplitude is originally antisymmetric with respect to $m_{\alpha}$ and $m_{\beta}$, this antisymmetry is not preserved by the expansion according to the hierarchy $m_{t} \gg m_{W} \gg m_{q}$. Similar arguments then indicate that the sur- viving result must involve $m_{\beta}$ so that we expect $d(d)$ $\propto m_{\beta} m_{i}^{2}$.

The above analysis gives no information about possible enhancements by factors of $m_{t}^{2}$ or logarithms. An explicit calculation is therefore necessary. The relevant amplitude consists of two sets of terms, one proportional to $\left(c_{\theta}^{2}\right.$ $\left.-s_{\theta}^{2}\right)\left(c_{\theta}^{2}+s_{\theta}^{2}\right)$, the other proportional to $\left(c_{\theta}^{2}-s_{\theta}^{2}\right) c_{\theta} s_{\theta}$. The first set is of a separate form as shown in Eq. (6) and canceled by the unitarity of the CKM matrix upon summing over the flavor pair $(j k)$. One or two terms in the second set are accidentally of the separate form and thus also canceled, but others are not. These latter terms produce the following leading contributions which are least suppressed by light quark masses:

$$
\begin{aligned}
d(u)= & +\frac{e G_{F}^{2}}{32 \pi^{4}} \widetilde{\delta} m_{b}^{2} m_{s}\left(c_{\theta}^{2}-s_{\theta}^{2}\right) c_{\theta} s_{\theta}\left(\left[\left(\frac{43}{18} Q_{d}\right)+0+0+\left(Q_{u}\left(\frac{1}{24}+\frac{1}{4} \ln \frac{m_{t}^{2}}{m_{W}^{2}}\right)+Q_{d}\left(-\frac{5}{72}-\frac{1}{6} \ln \frac{m_{t}^{2}}{m_{W}^{2}}\right)\right)\right]\right. \\
& \left.-\left[\left(Q_{u}\left(-\frac{5}{2}+\frac{\pi^{2}}{3}\right)-Q_{d}\right)+\left(-\frac{5}{4} Q_{u}+\frac{3}{2} Q_{d}\right)+\left(Q_{u}\left(-\frac{19}{3}+\frac{2 \pi^{2}}{3}\right)-\frac{1}{3} Q_{d}\right)+0\right]\right\} \\
= & +\frac{e G_{F}^{2}}{32 \pi^{4}} \widetilde{\delta} m_{b}^{2} m_{s}\left(c_{\theta}^{2}-s_{\theta}^{2}\right) c_{\theta} s_{\theta}\left[Q_{u}\left(\frac{81}{8}-\pi^{2}+\frac{1}{4} \ln \frac{m_{t}^{2}}{m_{W}^{2}}\right)+Q_{d}\left(\frac{155}{72}-\frac{1}{6} \ln \frac{m_{t}^{2}}{m_{W}^{2}}\right)\right], \\
d(d)= & -\frac{e G_{F}^{2}}{32 \pi^{4}} \widetilde{\delta} m_{b}^{2} m_{c}\left(c_{\theta}^{2}-s_{\theta}^{2}\right) c_{\theta} s_{\theta}\left[\left(-4 Q_{u}+3 Q_{d}\right)+\left(Q_{u}\left(5-\frac{2 \pi^{2}}{3}\right)+Q_{d}\left(-\frac{1}{2}+\frac{\pi^{2}}{6}+\frac{1}{2} \ln \frac{m_{b}^{2}}{m_{W}^{2}}\right)\right)\right. \\
& \left.+0+\left(Q_{u}\left(-\frac{1}{2}+\frac{\pi^{2}}{6}-\frac{3}{4} \ln \frac{m_{t}^{2}}{m_{W}^{2}}\right)+Q_{d}\left(-\frac{5}{4}+\frac{3}{4} \ln \frac{m_{t}^{2}}{m_{W}^{2}}\right)\right)\right] \\
= & -\frac{e G_{F}^{2}}{32 \pi^{4}} \widetilde{\delta} m_{b}^{2} m_{c}\left(c_{\theta}^{2}-s_{\theta}^{2}\right) c_{\theta} s_{\theta}\left[Q_{u}\left(\frac{1}{2}-\frac{\pi^{2}}{2}-\frac{3}{4} \ln \frac{m_{t}^{2}}{m_{W}^{2}}\right)+Q_{d}\left(\frac{5}{4}+\frac{\pi^{2}}{6}+\frac{3}{4} \ln \frac{m_{t}^{2}}{m_{W}^{2}}+\frac{1}{2} \ln \frac{m_{b}^{2}}{m_{W}^{2}}\right)\right] .
\end{aligned}
$$

Some explanations are in order. $\widetilde{\delta}$ is the usual rephasing invariant of CP violation [32], which is $c_{1} c_{2} c_{3} s_{1}^{2} s_{2} s_{3} \sin \delta$ in the original parametrization of the CKM matrix [6]. In the above formulas, only the largest terms are kept. The two terms in the braces for $d(u)$ originate from the top and the charm quarks, respectively; $d(d)$ is contributed totally by the top quark. The four terms in each square bracket of the first equalities for $d(u)$ and $d(d)$ arise from $W G, G W, W W$, and $G G$ exchanges in loops. We found no leading terms which are enhanced by $m_{t}^{2}$ or dilogarithms. The absence of $m_{t}^{2}$ enhancement is consistent with general arguments based on gauge invariance and naive dimensional analysis [33]. For numerical analysis, we take the following input parameters: $G_{F} \sim 1.2 \times 10^{-5} \mathrm{GeV}^{-2}, \quad \widetilde{\delta} \sim 5 \times 10^{-5}, \quad m_{t} \sim 175 \mathrm{GeV}, \quad m_{b}$ $\sim 4.5 \mathrm{GeV}, m_{c} \sim 1.5 \mathrm{GeV}, \quad m_{s} \sim 200 \mathrm{MeV}, m_{W} \sim 80 \mathrm{GeV}$. We obtain

$$
\begin{aligned}
& d(u) \sim-4 \times 10^{-34} \frac{s_{\theta}}{10^{-2}} e \mathrm{~cm}, \\
& d(d) \sim+6 \times 10^{-32} \frac{s_{\theta}}{10^{-2}} e \mathrm{~cm}, \\
& d(n) \sim+8 \times 10^{-32} \frac{s_{\theta}}{10^{-2}} e \mathrm{~cm} .
\end{aligned}
$$

These numbers are generally too small to be observable in the near future if there is no significant enhancement from long distance physics, but they are still much larger than the values in the SM [15],

$$
d(u) \sim-2 \times 10^{-35} \frac{m_{u}}{5 \mathrm{MeV}} e \mathrm{~cm},
$$




$$
d(d) \sim-8 \times 10^{-35} \frac{m_{d}}{10 \mathrm{MeV}} e \mathrm{~cm} .
$$

To summarize, we have considered the light quark and the neutron EDM's under the assumptions that the $C P$ source is still in the usual CKM matrix and that there is a small mixing of right-handed charged currents in the quark sector. We found that the EDM's arise already at two-loop order. The effect is of normal size in the sense that compared to new physics effects studied in the literature, it is small only because the $C P$ parameter $\widetilde{\delta}$ and the mixing angle $\theta$ are most stringently constrained in the current case, which bring down EDM's by a factor of $10^{7}$. The enhancement over the SM is also significant. This phenomenon caused by the left-right mixing was also observed in the decay $b \rightarrow s \gamma$ [34]. Finally, our study provides a good explanation of the result in the SM. There are two points which are responsible for the vanishing result at two-loop order. One is the unitarity of the CKM matrix, the other is the pure chirality structure of charged currents. This pure chirality structure diminishes further the nonvanishing result at three-loop order by introducing a small $u$ or $d$ quark mass to flip the chirality of the EDM operator.

Y.L. would like to thank the High Energy Physics Group of Michigan State University for its hospitality where part of the work was done. He is grateful to C.-P. Yuan for his interest and helpful discussions.
[1] For reviews, see S. M. Barr and W. J. Marciano, in CP Violation, edited by C. Jarlskog (World Scientific, Singapore, 1989), p. 503; X.-G. He, B. H. J. McKellar, and S. Pakvasa, Int. J. Mod. Phys. A 4, 5011 (1989).

[2] K. Abdullah et al., Phys. Rev. Lett. 65, 2347 (1990); E. D. Commins et al., Phys. Rev. A 50, 2960 (1994).

[3] K. F. Smith et al., Phys. Lett. B 234, 191 (1990); I. S. Altarev et al., ibid. 276, 242 (1992).

[4] P. G. Harris et al., Phys. Rev. Lett. 82, 904 (1999).

[5] R. Golub and K. Lamoreaux, Phys. Rep. 237, 1 (1994).

[6] M. Kobayashi and T. Maskawa, Prog. Theor. Phys. 49, 652 (1973).

[7] E. P. Shabalin, Yad. Fiz. 28, 151 (1978) [Sov. J. Nucl. Phys. 28, 75 (1978)].

[8] J. F. Donoghue, Phys. Rev. D 18, 1632 (1978).

[9] A. Czarnecki and B. Krause, Acta Phys. Pol. B 28, 829 (1997).

[10] Y. Liao and X. Li, Phys. Rev. D 60, 073004 (1999).

[11] I. B. Khriplovich and M. E. Pospelov, Yad. Fiz. 53, 1030 (1991) [Sov. J. Nucl. Phys. 53, 638 (1991)].

[12] M. J. Booth, Report EFI-93-01, hep-ph/9301293.

[13] E. P. Shabalin, Yad. Fiz. 31, 1665 (1980) [Sov. J. Nucl. Phys. 31, 864 (1980)].

[14] I. B. Khriplovich, Phys. Lett. B 173, 193 (1986); Yad. Fiz. 44, 1019 (1986) [Sov. J. Nucl. Phys. 44, 659 (1986)].

[15] A. Czarnecki and B. Krause, Phys. Rev. Lett. 78, 4339 (1997).

[16] J. Ellis, S. Ferrara, and D. V. Nanopoulos, Phys. Lett. 114B, 231 (1982); J. Polchinski and M. B. Wise, ibid. 125B, 393 (1983); M. Dugan, B. Grinstein, and L. Hall, Nucl. Phys. B255, 413 (1985); A. Sanda, Phys. Rev. D 32, 2992 (1985).

[17] Y. Kizukuri and N. Oshimo, Phys. Rev. D 45, 1806 (1992); 46, 3025 (1992).

[18] T. Ibrahim and P. Nath, Phys. Rev. D 57, 478 (1998); M. Brhlik, G. J. Good, and G. L. Kane, ibid. 59, 115004 (1999).

[19] KTeV Collaboration, J. Belz, hep-ex/9903025.

[20] J. Pati and A. Salam, Phys. Rev. D 10, 275 (1974); R. Mohapatra and J. Pati, ibid. 11, 566 (1975); G. Senjanovic and R. Mohapatra, ibid. 12, 1502 (1975); R. Mohapatra and D. Sidhu, Phys. Rev. Lett. 38, 667 (1977); R. Mohapatra and G. Senjanovic, ibid. 44, 912 (1980); X. Li and R. E. Marshak, Phys. Rev. D 25, 1886 (1982).
[21] K. S. Babu, B. Dutta, and R. N. Mohapatra, hep-ph/9905464; M. S. Chanowitz, hep-ph/9905478.

[22] Y. Fukuda et al., Phys. Lett. B 436, 33 (1998); Phys. Rev. Lett. 81, 1158 (1998); 81, 4279(E) (1998); 81, 1562 (1998).

[23] W. J. Marciano, hep-ph/9902332.

[24] M. B. Gavela et al., Phys. Lett. 109B, 215 (1982); I. B. Khriplovich and A. R. Zhitnitsky, ibid. 109B, 490 (1982); I. Bigi and N. G. Uraltsev, Zh. Éksp. Teor. Fiz. 100, 363 (1991) [Sov. Phys. JETP 73, 198 (1991)].

[25] Particle Data Group, R. M. Barnett et al., Phys. Rev. D 54, 1 (1996).

[26] G. Beall and A. Soni, Phys. Rev. Lett. 47, 552 (1981).

[27] For EDM's due to the mismatch between left and right CKM matrices or the new phase in $W_{L, R}^{ \pm}$mixing, see, for example, D. Chang, C.-S. Li, and T.-C. Yuan, Phys. Rev. D 42, 867 (1990); Atwood et al., Phys. Lett. B 256, 471 (1991); D. Chang, W.-Y. Keung, and J. Liu, Nucl. Phys. B355, 295 (1991). For EDM's due to Higgs boson exchanges in left-right models, see, for example G. Ecker, W. Grimus, and H. Neufeld, ibid. B229, 421 (1983); Phys. Lett. 153B, 279 (1985); M. Wakano, Prog. Theor. Phys. 72, 180 (1984); Phys. Lett. B 173, 163 (1986); D. Cocolicchio and G. L. Fogli, Phys. Rev. D 35, 3462 (1987); D. Chang et al., ibid. 46, 3876 (1992); for EDM's in supersymmetric left-right models: M. Frank, ibid. 59, 055006 (1999).

[28] L. F. Abbott, Nucl. Phys. B185, 189 (1981); Acta Phys. Pol. B 13, 33 (1982), and references cited therein.

[29] M. B. Einhorn and J. Wudka, Phys. Rev. D 39, 2758 (1989); A. Denner, G. Weiglein, and S. Dittmaier, Phys. Lett. B 333, 420 (1994).

[30] X. Li and Y. Liao, Phys. Lett. B 356, 68 (1995); A. Denner, G. Weiglein, and S. Dittmaier, Nucl. Phys. B440, 95 (1995).

[31] K. Fujikawa, Phys. Rev. D 7, 393 (1973).

[32] C. Jarlskog, Phys. Rev. Lett. 55, 1039 (1985); O. W. Greenberg, Phys. Rev. D 32, 1841 (1985); D.-D. Wu, ibid. 33, 860 (1986).

[33] Y. Liao, Y.-P. Kuang, and C.-S. Li, Phys. Rev. D 54, 3503 (1996).

[34] P. Cho and M. Misiak, Phys. Rev. D 49, 5894 (1994); K. S. Babu, K. Fujikawa, and A. Yamada, Phys. Lett. B 333, 196 (1994); T. G. Rizzo, Phys. Rev. D 50, 3303 (1994). 